\title{
Capacidades y recursos que influyen en las empresas femeninas
}

\author{
Skills and resourses that influence the business women \\ Capacités et ressources qui ont de l'influence sur \\ les entreprises féminines
}

\section{Diana M. Escandón Barbosa \\ dmescandon@javerianacali.edu.co \\ Calle 18 \# 118- 250 \\ Cali- Colombia}

Estudiante del Doctorado en Administración Universidad del Valle, Magister en Ciencias de la Organización, Universidad del Valle. Docente e Investigadora Universidad Javeriana, Departamento Gestión de Organizaciones.

Alberto Arias Sandoval aarias@javerianacali.edu.co Calle 18 \# 118- 250 Cali- Colombia

Doctor Economía y dirección de Empresas. Universidad Deusto. Director Educación continua y consultoría, Universidad Javeriana.

Artículo Tipo: de investigación Según Clasificación Colciencias

Fecha de recepción: 28 de enero de 2011 Fecha de corrección: 03 de junio de 2011 Fecha de aprobación: 22 de junio de 2011

\section{Resumen}

Este artículo tiene como propósito tratar de conocer las principales capacidades y los recursos que influyen en las empresas femeninas. Para lo cual se analizan los datos recopilados por GEM Colombia 2009, pero filtrando la base de datos y permitiendo utilizar solo lo relacionado con el género femenino. Dentro de los resultados se logró obtener un modelo consistente con la literatura sobre competitividad, destacando los recursos humanos, la innovación y la internacionalización. Sin embargo, no hay evidencia que explique cada una de estas variables por género y mucho menos la relación con competitividad. Para lo cual, los autores plantean una primera aproximación y que puede generar tendencias investigativas al respecto. La metodología aplicada en este articulo, es un sistema de ecuaciones estructurales, mediante el paquete estadístico SPSS Amos 18, que permite generar un esquema de relaciones entre las variables, $y$, acercarse a las tendencias de modelos en las explicaciones de las ciencias sociales.

Palabras clave: competitividad, innovación, internacionalización, recurso humano, recursos y capacidades. 


\section{Skills and resourses that influence the business women}

\section{Capacidades y recursos que influyen en las empresas femeninas}

\section{Capacités et ressources qui ont de l'influence sur les entreprises féminines}

\author{
Capacidades y recursos que influyen en \\ las empresas femeninas
}

\section{Skills and resourses that influence the business women}

\begin{abstract}
This article is aimed at trying to get to know the main competitive factors within companies created by Colombian women for which are analyzed data collected by GEM Colombia 2009, but filtering the database and allowing to use only the related to the female gender. Within the results will be able to obtain a model consistent with the literature on competitiveness, emphasizing human resources, innovation and internationalization. However, there is no evidence that explain each of these variables by gender and much less the relationship with competitiveness. For which, the authors pose a first approach and that can generate investigative trends in this regard. The methodology used in this article is a system of structural equations, through the statistical package SPSS Amos 18, which allows you to generate an outline of relations between the variables, and closer to the trends of models in the explanations of the social sciences.
\end{abstract}

Keywords: competitiveness, innovation, internationalization, human resources, resources and capabilities.

\section{Résumée}

Cet article a pour objectif d'essayer de connaître les principales capacités et les ressources qui ont de l'influence sur les entreprises féminines. Pour cela on fait l'analyse des données recueillies par GEM Colombia en 2009, en filtrant la base de données pour permettre l'utilisation de l'information en relation avec le genre féminin. Parmi les résultats, on a pu obtenir un modèle cohérent avec la littérature sur la compétitivité, en remarquant les ressources humaines, l'innovation et l'internationalisation. Néanmoins, il n'y a pas d'évidence qui explique chacune de ces variables selon le genre, et encore moins, la relation avec la compétitivité. En vue de cette impasse, les auteurs proposent une première approximation qui peut générer des tendances de recherche. La méthodologie appliquée sur cet article repose sur un système d'équations structurelles, à partir du paquet statistique SPSS Amos 18, qui permet la création d'un schéma des relations entre les variables, et un rapprochement aux tendances des modèles dans les explications des sciences sociales.

Mots clef: compétitivité, innovation, l'internationalisation, des ressources humaines et de capacités. 


\section{Capacidades y recursos que influyen en las empresas femeninas}

\section{Introducción}

La noción de género ha hecho que se escriba gran cantidad de estudios desde diferentes perspectivas a lo largo de las últimas décadas, entre las que se destacan investigaciones en: diferencias simbólicas en las que se difieren los espacios masculinos y femeninos (Saco, 1992; Craig, 1992); diferencias al interior de las organizaciones en términos de contratación y promoción (Grant y Tancred, 1992), la división genérica del trabajo (Hunt, 1980; Armstrong y Armstrong, 1990) entre otras.

Estas diferentes investigaciones, buscan que la noción de género transcienda de las diferencias físicas o corporales en los seres humanos, y logre explicar la diferencias sicológicas, sociales y culturales, y que, son susceptibles de modificación a través del tiempo (Mertens, I. (2002)). Estas diferencias de género, logran generar una clasificación y valoraciones sociales, en términos de actividades, tareas y responsabilidades, es decir, superan la mera división en términos de sexo y transciende al contexto, es decir, "el género debe redefinirse y reestructurarse en conjunción con la visión de igualdad política y social que comprenda no solo el sexo, sino también la clase y la raza" (Scott, 1990).

Sin embargo, se debe considerar que ninguno de los roles ejercidos por hombres o por mujeres son de carácter natural o frutos de la naturaleza, sino que, por el contrario, son creaciones humanas, que nacen a partir de la concepción sociocultural, y que permiten relacionar comportamientos, actitudes y sentimientos como masculinos o femeninos. (Braidotti, 1991; Murillo, 2000; Beltrán et al. 2001). Estos roles pasan por un proceso de socialización y son incluidos en el imaginario y en la identidad de cada una de las personas que los adoptan, por lo tanto, logran crear una naturalización de roles por género (Bosch, 2001).

Estas divisiones naturalizadas, son consideradas como coercitivas y condicionan rol de la mujer en la sociedad, pues, esta pluralidad de roles femeninos: reproducción, matrimonio y cuidado de los niños, tiende a ocupar gran parte del tiempo y evitar incursionar en el mercado laboral o mentalizarla a abandonar sus actividades por fuera del hogar y lograr mantener la armonía en la familia y poder ocuparse de actividades complementarias que no rivalicen con los de los hombres (Shaktini, 1985).

Sin embargo, gran cantidad de investigaciones, especialmente de tipo empírico, evidencian la tendencia creciente de empresas creadas por mujeres, disminuyendo la brecha existente con el número de empresas constituidas por hombres, implicando una disminución, probable, de dichas divisiones naturalizadas, la incursión de las mujeres en el rol empresarial, mayores posibilidades de educación de las mujeres y por ende, el desarrollo de capacidades y conocimientos, la mayor incorporación de la mujer en el mercado laboral, la dificultad, en algunos casos, de ser absorbidas por dicho mercado o tener un empleo estable, la alta participación del sector servicios, debido a sus bajas restricciones de entrada (Guzman, J y Rodríguez, M; 2008).

Sobre las características de las empresas creadas por mujeres, la literatura es realmente baja, pero se logran resaltar algunos aspectos como: empresas de bajo tamaño, baja tasa de supervivencia empresarial, empresas con propiedad compartida, casi siempre de tipo familiar; dentro de las principales motivaciones para crear su propia empresa está la necesidad de compatibilizar su trabajo con el rol dentro del hogar. (Marlow, 1997, Boden y Nucci, 2000; Guzmán, J. y Rodríguez, M. 2008). Otros estudios afirman que la constitución de empresas por parte de mujeres se ve afectada por rasgos de personalidad, como baja propensión al riesgo, alta necesidad de logro, personalidad poco proactiva, (Ljunnggren y Kolvereid, 1996, Guzmán, J. y Rodríguez, M., 2008). Y por tanto estas características limitan posibilidades como entrar a nuevos mercados, creación de un portafolio más amplio de productos, entre otras posibilidades que se pueden suscitar en la actividad empresarial.

Algunas investigaciones, que logran analizar los factores de éxito de las empresas creadas por mujeres, (Guzmán, J. y Rodríguez, M., 2008, Gatewood, Shaver y Gartner,1995; Buttner y Moore, 1997) resaltan, la necesidad de desarrollo profesional, de autorrealización, de mantener el equilibrio laboral-familiar, superación de 
aspectos desmotivantes como salario y falta de desafíos profesionales.

\section{Teoría de los recursos y capacidades}

Una de las perspectivas dentro del campo del management estratégico que ha recibido gran atención en los últimos años es la denominada teoría de recursos y capacidades. Esta perspectiva se caracteriza por considerar que son los recursos y capacidades distintivas de las empresas, las que permiten generar condiciones para enfrentar una competencia y así lograr un exitoso desempeño económico. Por medio de esta perspectiva se contempla la competitividad desde el punto de vista de los recursos de las empresas que permiten generarla, visión que se complementa con el planteamiento de Porter, sobre el hecho que son las empresas las que compiten en los mercados y es en ellas en las que recae la responsabilidad de generar mecanismos que les permitan hacer un uso eficiente de sus recursos para poder enfrentar la competencia en el sector en el cual se encuentran insertas.

Uno de los autores pioneros sobre el tema de los recursos de las empresas como herramienta para el fortalecimiento competitivo de la empresa es Birger Wernerfelt, quien publicó un artículo en la revista Strategic Management Journal, en el año 1984 con el nombre de "A Resource based View of the Firm"; el cual tiene como propósito, en palabras del autor, desarrollar una herramienta para el análisis de los recursos de una firma y así tomar en cuenta algunas opciones estratégicas que permitan a la empresa consolidar su posición en el mercado. Por otra parte, cabe señalar que este autor enfatiza en la relación entre rentabilidad y los recursos de la empresa.

La teoría de los recursos brinda una definición de empresa como una recopilación no repetible de capacidades y recursos que no se pueden comprar ni vender libremente en el mercado (Conner, 1991; Rumelt, 1987; Wernerfelt, 1984). Los recursos pueden clasificarse como tangibles e intangibles, siendo los primeros aquellos activos que posee la empresa y se pueden detectar y medir como las instalaciones, la maquinaria, las materias primas, el capital, entre otros, mientras que los recursos intangibles no tienen soporte físico y dependen en su mayoría, principalmente, del conocimiento y la información, estos son las patentes de propiedad de la empresas, la(s) marca(s), también se consideran la cultura de la empresa, las redes personales y organizaciona- les, el Know-how de los trabajadores, asesores, proveedores y distribuidores; se podría decir que los recursos intangibles son originados por el recurso humano y recursos del capital de la empresa (Hall, 1992; Barney, 1991; Wernerfelt, 1984). Las capacidades son el resultado de la acción de la orientación para movilizar los recursos a través de la creación de un sistema de prácticas de la organización que permite la interacción entre estos y además, son las encargadas de la creación de valor dentro de la empresa (Cuervo, 1995; Grant, 1991).

Los recursos y capacidades comprenden tres ideas fundamentales: las organizaciones son diferentes una de otra debido a los recursos $y$ capacidades que posean en un momento determinando, sumado a la heterogeneidad de la mismas. Adicionalmente, aparece otro concepto denominado movilidad imperfecta el cual se refiere a que la disponibilidad de los recursos y capacidades no es igual para todas las empresas. De acuerdo a lo anterior es la heterogeneidad y la movilidad imperfecta las que explican el rendimiento financiero de las empresas pertenecientes a una misma industria (Ventura, 1996; Peteraf, 1993; Barney, 1991).

El recurso humano incluye todo tipo de conocimiento y capacidades que obtiene o desarrolla un individuo. (Becker, 1964). Sin embargo, la literatura existente afirman no encontrar diferencias sustanciales entre los niveles educativos de las empresarias y los empresarios, es decir, el género, no constituye una diferencia, a priori, en el desarrollo empresarial, (Birley, Moss y Sander, 1987; Fisher et al. , 1993, Díaz y Jiménez, 2004), algunos otros estudios contradicen estos resultados (Srinivasan, Woo y Cooper, 1994), y se argumentan en el tipo de formación adquirida por la mujeres, pues, tiene una alta tendencia hacia las humanidades, y no hacia los negocios 0 la administración (Cliff, 1998).

En consecuencia, se puede ver al recurso humano como elemento integrador y generador de habilidades para la utilización eficiente de los demás componentes de la ventaja competitiva (Diéguez et al.2007; Sisson y Storey, 2000; Snell y Dean, 1992).

Se debe resaltar que, aunque existen diferentes formas de medir el recurso humano, desde la teoría de recursos y capacidades como la edad, experiencia, entre otras, para este estudio se utiliza como parámetro de medición 
la acumulación de conocimientos por medio de la educación, pues logra generar un gran vínculo de interrelación con el recurso innovación, y al mismo tiempo, permite considerarlo no sólo como un recurso sino como una capacidad; es decir, los individuos más educados están en mejores condiciones de resolver problemas e incentivan más la innovación, haciendo que mejore el nivel de eficiencia y de consecución de las ganancias de la organización (Nelson y Phelps, 1996; Gill, 1989).

Tomando al emprendedor (a) como un recurso humano fundamental a la hora de crear empresa, los conocimientos que haya adquirido hacen que sus capacidades aumenten y tengan un efecto positivo en el negocio; para diversos autores la variable nivel de educación es una variable proxy de la cantidad de conocimientos que posee el individuo (Gamero y Lassibille, 2008). Un mayor capital humano aumenta la productividad del fundador de la empresa, que se traduce en mayores beneficios (Bates, 1985). Mayor productividad significa que el fundador es más eficiente en la organización y en la gestión del proceso de producción o es capaz de atraer a más clientes y nuevo capital de los inversionistas (Bruderl et al. 1992).

Estudios complementarios logran encontrar que no sólo la inversión en capital humano, específicamente en capacitación, tiene impacto en la competitividad de la empresa que lo desarrolla, sino que se traslapa al sector, región o localidad en donde opera, a través de la difusión de conocimientos, interacción o cooperación empresarial y movilidad de los trabajadores. (Cooke et al. 1997; Howells, 1999; Carlsson, Jacobsson y otros, 2002; lammarino, 2005). Estos factores solo pueden ser analizados mientras la interacción entre las empresas pueda ser de tipo frecuente o de cercanía geográfica, asegurando la verdadera transferencia de conocimiento (Cantwell y lammarino, 2003; Cantwell y Molero, 2003; Malmberg et al, 1996), es decir, la participación en redes, se constituye en un elemento principal para el desarrollo empresarial (Casrud, Gaglio y Olm, 1986).

Algunas investigaciones muestran que la conformación de redes, no difiere mucho, al ser discriminada por género. Sin embargo, las mujeres, tienden a formar redes mucho más pequeñas que los hombres empresarios, limitando sus posibilidades de explotar este tipo de recurso, y no permitiendo generar una verdadera disminución de costes. (Diaz y Jiménez, 2004). Esto puede ser fuertemente atribuible, a la necesidad de las mu- jeres de mantener el equilibrio entre sus labores empresariales y su rol en la familia, que no permite que dediquen el suficiente tiempo para la consolidación de redes empresariales. (Moore y Buttner, 1997). Otro tipo de hallazgos, es la creación de redes, que está compuesta mayoritariamente por familiares cercanos, que al mismo tiempo pueden ser fuentes de financiación. (Renzulli et al. ,2001)

Por otra parte, una capacidad de gran importancia para el desarrollo competitivo de las empresas creadas por mujeres es la innovación, que puede verse por medio de la consecución de capacidades estratégicas relacionadas con tecnologías y uso de recursos, capacidad de organización (coordinación de los recursos), capacidad de implementación u operativa (hacer las cosas bien), capacidad de adaptación al cambio o aprendizaje, capacidad de generación de incentivos o motivación. Estas capacidades permiten dar a las organizaciones, en términos de Barney (1991) una ambigüedad causal, o, según Grant (1991), una replicabilidad imperfecta, y así obtener o mantener la ventaja competitiva.

Aunque no existen estudios empíricos que analicen la innovación discriminada por género, si se afirma que la innovación tiene un impacto positivo en la competitividad, especialmente, cuando se desarrollan capacidades de relación o de participación en redes de cooperación, recursos humanos calificados e involucrados en procesos de aprendizaje continuo para poder realizar innovaciones constantes y habituales (Rodeiro y López, 2007).

Sin embargo, la innovación es una condición necesaria pero no suficiente para alcanzar la competitividad, debido a que la competencia en la economía mundial se da entre sistemas productivos al interior de los cuales actúan las empresas, donde se encuentra el apoyo financiero, de tecnología, de subsidios. En efecto, la competitividad es una variable multifactorial que se compone de formación empresarial, prácticas administrativas, laborales y productivas, la innovación (interna o externa) y el progreso tecnológico (Corona Treviño, 2002).

La innovación tiene dos efectos en una empresa: el incremental y el radical, ambos están en función de lo novedosos que son los conocimientos y del nuevo producto o servicio. Bajo este enfoque, la innovación es incremental cuando se ofrece un nuevo producto o servicio apoyado en el conocimiento ya existente, mientras que la in- 
novación radical genera nuevo conocimiento para crear un nuevo producto, y vuelve en conocimiento existente obsoleto (Afuah, 1999). Es importante resaltar que la mayoría de innovaciones son sólo de tipo incremental, y por tanto, la posibilidad de mantener una ventaja competitiva tiende a ser sólo en el mediano y corto plazos, pues la competencia tiene mayores posibilidades de adquirir o replicar este tipo de innovación.

La capacidad de innovación de una empresa, al mismo tiempo, puede propiciar la incursión en nuevos mercados, que, a su vez, puede asegurarle un mejor aprendizaje organizacional, y un mayor posicionamiento en el mercado. Valenzuela (2000) sostiene que "el comportamiento exportador puede ser conceptualizado como una respuesta estratégica condicionada a la dotación de recursos y capacidades de la empresa". El modelo plantea que la internacionalización surge como una acción de tipo estratégico de la empresa para lograr mejorar su nivel de utilidades o posicionamiento y sólo puede ser logrado si se maximiza la capacidad exportadora por medio de la utilización adecuada de los recursos internos que posee la empresa tales como capacidades de marketing, organizacionales, apertura exportadora, tecnología, etc.

\section{Metodología y variables}

Para este trabajo se hace un análisis de los datos relacionados con algunas variables del informe GEM Colombia 2009. Dichos datos fueron recopilados por medio de una encuesta realizada por el Centro Nacional de Consultoría (CNC) y que se aplicó telefónicamente a través de 1600 cuestionarios en las cinco principales ciudades capitales (Bogotá, Medellín, Cali, Barranquilla y Bucaramanga) y en tres ciudades capitales de departamento (Santa Marta, Cúcuta y Pasto) y 400 cuestionarios cara a cara en 23 municipios, de áreas rurales, seleccionados aleatoriamente con poblaciones inferiores a 10000 habitantes ${ }^{1}$.

Para analizar los datos y cumplir con los objetivos trazados en este estudio, primero se realiza estadística descriptiva teniendo en cuenta sólamente la caracterización para el género femenino. Se analizan variables como tipo de involucramiento en el emprendimiento por oportunidad, ingreso total del negocio, nivel educativo, inicio del negocio como alternativa de carrera, Consideración del producto como nuevo, tipo de tecnología usada, actividad económica de la empresa y nivel de intensidad de la exportación, si la hay. Para este análisis se utiliza el programa SPSS en su versión 18.0.

Luego, se genera un modelo de ecuaciones estructurales, donde se precisan las diferentes interacciones existentes entre las variables latentes y los parámetros. Este tipo de modelos permite analizar relaciones recíprocas y simultáneas presentes entre diferentes variables que miden un constructo (como la calidad del servicio), mientras toma en cuenta el error de medida (Saurina y Coenders, 2002).

Mientras el modelo de medida especifica las relaciones que guardan los factores o variables latentes con sus respectivos indicadores (Jöreskog, 1969), la parte estructural especifica las relaciones direccionales de las variables latentes (o dimensiones como en este caso) entre sí. Los resultados del modelo se analizaron con el programa AMOS 18 , que se validan mediante la técnica del análisis factorial confirmatorio, mediante el cual se tiene un control completo sobre la especificación de los indicadores de cada factor (variable latente). Además, permite un test estadístico de calidad de ajuste para la solución confirmatoria del factor propuesta.

\subsection{Definición de las variables del modelo}

Como parte del proceso de internacionalización de las empresas, se tuvieron en cuenta las siguientes variables creadas a través de la Tasa nueva de actividad empresarial (TEA). Esta tasa mide el emprendimiento durante el inicio de la actividad hasta tres años y medio de vida de la empresa.

* Internacionalización: se compone de niveles, iniciando con el más alto (75\%-100\%), seguido por un nivel medio (25\%-75\%) y terminando con el más bajo (1-25\%). Aunque existe la posibilidad de evaluar un nivel diferente.

* Actividad Económica: los sectores productivos donde las nuevas empresas pueden desarrollarse son el sector extractivo (agricultura, forestal, pesca, minería), el sector de transformación (manufactura, ventas al por mayor, construcción), el sector financiero

1. En este trabajo participaron la Universidad de Los Andes, Universidad ICESI, Universidad del Norte y la Pontificia Universidad Javeriana de Cali. 
y el sector de servicios de consumo (bares, hoteles, restaurantes, comercio al por menor, salud, educación, servicios sociales, recreación).

* Motivación: para las emprendedoras, el tipo de involucramiento que tengan para iniciar un negocio por oportunidad es medido a través de la mayor independencia que puedan lograr, el aumento o la conservación del ingreso personal, la continuidad del negocio familiar, etc.

* Innovación: se refiere al nivel de innovación en el producto de la empresa, expresando que todos, algunos o ningún cliente considera el producto nuevo.

* Competencia: mide el nivel de innovación de la empresa y muestra si tiene muchos, pocos o ningún competidor.

* Nivel Socioeconómico: presenta los 6 niveles de estratificación económica que existe en Colombia.

* Nivel Educativo: mide el recurso humano que pueda tener la empresa. Su clasificación es sin educación, nivel primario, secundario, tecnológico, universitario y graduado.

* Nivel Tecnológico: identifica el recurso tecnológico de una empresa nueva.

* Reconocimiento Social: identifica si ser empresario constituye una opción de carrera para los empresarios, y la percepción social.

Por otra parte, se creó la variable latente llamada Competitividad, la cual es un componente clave para determinar la relación existente con internacionalización a través de las exportaciones de las empresas. Se construye sobre los rendimientos financieros de las empresas creadas por mujeres.

\subsection{Construcción del Diagrama de Secuencias de Relaciones Causales}

\section{Elementos del diagrama}

* Variables Endógenas: estas son explicadas por una o más variables del modelo y también sirven para predecir el comportamiento de otras variables endógenas. Pertenecen a esta categoría innovación en el producto, recurso humano, nueva tecnología, competidores, nivel socioeconómico, tipo de oportunidad, actividad económica, intensidad de la exportación y modo de expansión del mercado.

* Variables Exógenas: son variables independientes que están determinadas fuera del modelo, es decir, no se encuentran explicadas por alguna variable observable. Sólo mantienen relaciones de causalidad con los constructos endógenos. Pertenece a esta categoría la variable latente competitividad.

* Flechas: las flechas en una sola dirección indican relaciones de causalidad directa de una variable a otra, mientras que las flechas de doble dirección indican correlaciones entre las variables.

\section{Resultados y discusión}

\subsection{Análisis descriptivo de variables individuales}

En cuanto a los atributos personales que prevalecen en las mujeres emprendedoras encuestadas, se resalta que, menos del $7.0 \%$ pertenecen a los niveles socioeconómicos altos ( 5 y 6), generando un alto predominio de niveles medio-bajos, especialmente del estrato dos, donde se concentra cerca del $40 \%$, dentro del cual las mujeres de 18 a 27 años representan $22.9 \%$ de los casos, el rango de 28 a 39 años el $29.4 \%$, entre 40 y 50 años el $27.1 \%$ y superiores a 51 años el $20.7 \%$. Esta tendencia genera un predominio de esfuerzos de emprendimientos por necesidad representado el $92.1 \%$ del total de casos, sólo el $7.9 \%$ es catalogado como por oportunidad. En el caso de los emprendimientos por necesidad, el $38.3 \%$ pertenecen al nivel socioeconómico dos, seguido por el estrato tres $(28,2 \%)$ y el nivel $1(10,9 \%)$.

Por otra parte, el $\mathbf{2 4 . 6 \%}$ de las encuestadas, tienen como nivel educativo la finalización de la secundaria; y solo el $3.7 \%$ se ha graduado del pregrado. Esta caracterización educativa, genera una alta tendencia a niveles de ingresos relativamente bajos dentro del hogar, pues el $71.8 \%$ logran obtener menos de $\$ 867.000$ (menos de 2 SMLV) y sólo el $12.2 \%$ de la población femenina tiene ingresos superiores a $\$ 1.300 .000$ (más de 3SMLV).

Estos bajos niveles de ingresos, generan una percepción por parte de las mujeres de la nece- 
sidad de crear su propia empresa y concebirla con altas expectativas por constituirse en una buena opción de carrera, dada el grado de flexibilidad ofrecida y el equilibrio que puede generar para desarrollar las otras facetas de la vida (familia, hijos, educación, entre otros). Este tipo de necesidades personales, se constituye en un objetivo primordial y hace que las mujeres se preocupen menos por obtener altas rentabilidades, debido a esto, las mujeres dueñas de empresas pequeñas tuvieron menos beneficios. Otros estudios, han encontrado este mismo fenómeno y se asocia a que la productividad medida por la razón de beneficio es más baja en las mujeres con relación a los hombres debido a los obstáculos enfrentados en sectores de educación, roles del hogar y la creación de redes (De Martino y Barbato, 2003; Fasci y Valdez, 1998).

Otro aspecto relevante es que el tipo de productos que salen al mercado, resultado de los esfuerzos de las emprendedoras, tienen bajo nivel de innovación (27,5\%), lo cual puede ser explicado por la tendencia a una proliferación de emprendimientos por necesidad, es decir, la mayoría de productos asociados al emprendimiento no están generando un valor agregado que permita consolidar a estas empresas dentro del mercado y asegurar rendimientos superiores.

Estos factores, se agregan a la escasa incorporación de las nuevas tecnologías, tanto de las nuevas empresarias (empresas nacientes) como de las ya establecidas. En el caso de los nuevos negocios, la mujer podría incorporar un mayor porcentaje de las nuevas tecnologías entre sus procesos ( $58 \%$ ). Sin embargo, este tipo de tecnología es el tipo de minoría dentro de las organizaciones, que representan el $24,6 \%$ en el caso de las mujeres y el $16,9 \%$ en los hombres. Mientras que la vieja tecnología, la que tiene más de 5 años, tiende a ser mayoría con un $44,9 \%$ y un $47,6 \%$ del emprendimiento femenino y masculino, respectivamente.

En el caso de empresas ya establecidas, la situación tiende a ser más preocupante, ya que los porcentajes de uso de la tecnología que superan los 5 años, alcanzan un nivel superior al $59 \%$ en los diferentes géneros, es decir, pierde cuota tanto la nueva tecnología como la tecnología intermedia.
Los bajos niveles de tecnología incorporada y la baja innovación de los productos en las empresarias encuestadas se pueden ver reflejados en una baja intensidad de exportación, dado que el $81,5 \%$ y el $77,6 \%$ de las mujeres y los hombres, respectivamente, afirman que no exportan. Este alto nivel se explica por la baja incorporación de valor agregado a los productos y la dificultad de entrar a los mercados internacionales. De la misma manera, puede ser un indicador cultural, de no hacer un esfuerzo de posicionamiento fuera del país, incluso muchas empresas pequeñas, ni siquiera transcienden más allá de su localidad o ciudad en la que se establecen inicialmente.

\subsection{Análisis de las variables del modelo}

La siguiente gráfica enseña el modelo finalmente construido donde se pueden apreciar las relaciones que existen con respecto a la variable de competitividad (Gráfica 1).

Una vez se ha establecido el modelo, se procede a evaluar su ajuste global con una o más medidas de calidad de ajuste. La calidad del ajuste mide la correspondencia entre la matriz de entrada real, con la que se predice a través del modelo. La primera medida que se estimó fue el ratio de verosimilitud chi-cuadrado, que tuvo un nivel de significación estadística igual a 13. Sugiriendo evidencia de bajas diferencias significativas entre las matrices efectivas y previstas.

Frente a estos resultados, es necesario aclarar que la literatura llama constantemente la atención sobre la necesidad de interpretar con cuidado este estadístico, dado que ha demostrado ser altamente sensible al tamaño de la muestra. A mayor tamaño de muestra (\$100), cualquier diferencia entre el modelo previsto y los datos obtenidos, se magnifica (Hair et al., 1998).

Debido a lo anterior, se decidió evaluar el ajuste global del modelo utilizando medidas absolutas y medidas incrementales. Las primeras determinan el grado de relación que presenta la matriz estimada por el modelo y la matriz de datos inicial. Las segundas comparan el modelo propuesto con el modelo independiente, el cual muestra un nivel nulo de asociación entre las variables ${ }^{2}$. Los resultados obtenidos en la evaluación se presentan en la siguiente Tabla 1.

2. Los índices de ajuste incremental (índice de ajuste normado-NFI- e índice de ajuste no normado -NNFI) comparan el modelo propuesto con un modelo de referencia (modelo nulo). Mientras más cerca de 1 se encuentren, mejor ajuste del modelo reflejan. Las medidas de ajuste de parsimonia no se extrajeron debido a que éstas son más útiles cuando se trata de comparar modelos rivales, caso que no era el nuestro. 


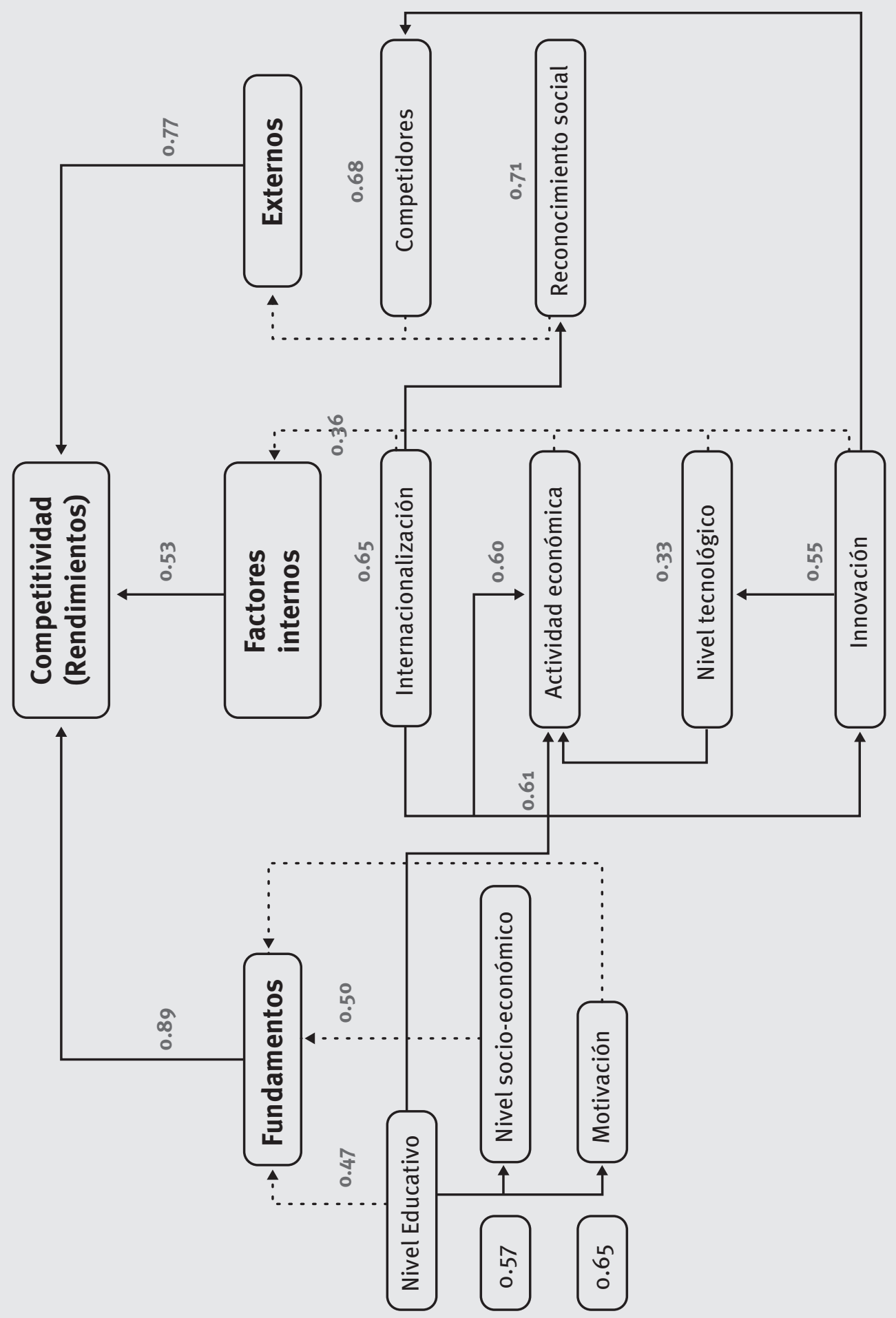

En el caso de índices incrementales como NFI y TLI podemos ver buenos resultados, significando que nuestro modelo es opuesto al modelo nulo, que indica falta absoluta de asociación en las variables. Para este trabajo, vemos que se logra obtener altos niveles de aceptación (o,98 y 0,93 respectivamente). Por otra parte, el CFI, IFI y RFI toman niveles cercanos a 1, tomando o,98, 0,90 y 0,97 respectivamente.

Las medidas de desempeño arrojadas por el modelo estructural, poseen índices altamente 
Tabla 1. Indicadores de Bondad de Ajuste

\begin{tabular}{|c|c|c|}
\hline Medidas de Ajuste Absoluto & Modelo Estructural & Niveles de Aceptación \\
\hline Chi-cuadrado & & \\
\hline Grados de libertad & 13 & Cercanos a chi- Cuadrado \\
\hline Valor p & 8 & Niveles iguales o superiores 0,05 \\
\hline Error de Aproximación Cuadrático & 0.05 & $0.02<0.05$ \\
\hline medio RMSEA & 0.025 & \\
\hline
\end{tabular}

Fuente: elaboración propia.

aceptables para validar su uso en este análisis. Sin embargo, la idea del modelo es comprobar los factores que inciden en el éxito de las empresas conformadas por las mujeres colombianas generando coeficientes de causalidad relativamente altos y que le dan viabilidad a la utilización de este modelo.

Otro tipo de análisis realizado es el Alpha Cronbach, que analiza la rigurosidad y la consistencia interna del modelo, logrando para este estudio un nivel de 0.93 , el cual supera los niveles mínimos recomendados para estudios confirmatorios (o.80). Adicionalmente, se cumple con la propiedad de parametrización escueta, es decir, se logra obtener el mínimo número de ítems con alto nivel de información relevante.

\subsection{Resultados Generales}

Según los resultados que se presentan en el informe del GEM Colombia 2009, se observa que los nuevos emprendimientos femeninos (menos de 3.5 años) están relacionados con los bajos niveles de ingresos de las mujeres que los crean. Esto genera la percepción que las mujeres asumen este reto por la necesidad de generarse su propio ingreso y con altas expectativas de que este sea su opción de vida laboral, dada el grado de flexibilidad que ofrece y el equilibrio que puede generar para desarrollar las otras facetas de la vida (familia, hijos, educación, entre otros). Este tipo de necesidades personales, se constituyen en su objetivo primordial y hace que las mujeres se preocupen menos por obtener altas rentabilidades. (Fasci and Valdez, 1998; R DeMartino, R Barbato, 2003).

Como resultado de este análisis, este estudio concluye que la internacionalización de las nuevas empresas creadas por mujeres colombianas, es explicada por la innovación, el tipo de actividad económica y el modo de expansión de la empresa. No es extraño afirmar que productos con alta calidad y que sean nuevos para el consumidor tienen mayores ventajas al entrar en nuevos mercados; es de resaltar que el sector económico donde las mujeres crean más empresas es en actividades orientadas al consumo. Sin embargo, la variable tecnología influye negativamente en las exportaciones tempranas, al parecer estos productos innovadores no están respaldados por nuevas tecnologías.

Otro resultado interesante es que la mayor educación de las mujeres es explicado por el nivel socioeconómico al que pertenecen, es claro que las mujeres que viven fuera de los niveles de pobreza tienen un nivel de educación superior que le da más habilidades y destrezas a la hora de crear su propia empresa. Así las mujeres con más educación, son las que logran que su empresa se expanda en su mercado, siendo más competitivas, lo cual se refleja en la mayor productividad de estas. Lo anterior se ve reflejado en la calidad del producto, el nivel de innovación y la manera como se hace efectivo el servicio.

La innovación de las empresas femeninas, se ve influenciada por el nivel de competencia, a mayor sea la cantidad de competidores, más innovación desarrolla en la empresa, pero esto está mediado por el mayor interés de estas mujeres por lograr su independencia económica.

\section{Conclusiones}

La variable internacionalización es explicada positivamente por la innovación y el tipo de actividad. No es extraño afirmar que productos con alta calidad y que sean nuevos para el consumidor tienen mayores ventajas al momento de ofrecerlos; también cabe resaltar que el sector económico donde las mujeres crean más empresas es en actividades orientadas al consumo. Por el contrario, la variable tecnología influye negativamente en la intensidad de las exportaciones debido a que las mujeres no indican niveles de nueva tecnología en las nuevas empresas. 
Por otra parte, la variable Recurso Humano es impactada positivamente por el nivel socioeconómico, lo cual podría dar a entender que entre mayores oportunidades tengan las mujeres de vivir fuera de los niveles de pobreza, así mismo, su nivel de educación será cada vez mayor, generando habilidades y destrezas que son atractivas a la hora de la contratación laboral. De la misma manera, el capital humano influye positivamente en el modo como la empresa expande su mercado y atrae clientes; esto se debe a que cuando existe personal altamente calificado o con estudios superiores, la organización se hace cada vez más fuerte, ágil y con mejores proyecciones. Lo anterior se ve reflejado en la calidad que tenga el producto, el nivel de innovación y la manera como se hace efectivo el servicio. También, la empresa podrá crecer en un sector productivo específico con trabajadores especializados para ello, es decir, los trabajadores asumirán responsabilidad organizacional en la medida en que todo su esfuerzo, conocimiento y dedicación lo compartan para el bien común. En términos de la inversión en niveles de educación, se genera un efecto positivo sobre el nivel de los rendimientos de este tipo de organizaciones. Es decir, que si las mujeres emprendedoras logran obtener un alto nivel de formación podrían contribuir en mayor proporción a la competitividad de su empresa.

La variable Innovación presenta relaciones causales positivas a partir de la cantidad de competidores que tenga la empresa y el tipo de oportunidad que tomaron las mujeres para crear nuevas empresas, teniendo en cuenta que lo que buscan es mayor independencia económica. Aunque las inversiones en actividades de innovación, en promedio, son muy reducidas (0,05\% del PIB) si se compara con otros países, situación que puede determinar la tendencia a generar innovaciones de tipo incremental y no de tipo radical. Esta situación limita los resultados en términos de competitividad $y$, por ende, plantea la necesidad de mejorar los niveles de inversión en recursos humanos y tecnológicos para fomentar el desarrollo de nuevos productos $y / 0$ procesos. Una posible solución para mejorar los niveles de innovación y, por ende, la competitividad, es por medio del desarrollo de capacidades de relación o de cooperación, que permita el intercambio de información, de recursos para el desarrollo compartido de capacitación tecnológica, financiación, entre otros recursos o capacidades que pueden llegar a consolidar una ventaja competitiva.
Este estudio permite sensibilizar con respecto al género, reconociendo la existencia de diferentes roles, pero altamente correlacionados (mujer, madre, empresaria, etc.), haciendo necesario la creación de políticas y programas específicos, y que generen un balance entre sus papeles socialmente aceptados.

Por otra parte, es necesario reconocer que este estudio no logra incluir variables que reporten la existencia de otros recursos o capacidades presentes en las diferentes organizaciones, debido, principalmente, a la limitación de la base de datos utilizada. Sin embargo, no se desconoce la relevancia de recursos y capacidades relacionadas. También esta investigación presenta interesantes datos que brinda a las autoridades de políticas públicas colombianas, permitiéndoles crear nuevos programas de apoyo y llegar a tomar decisiones para fomentar y consolidar los emprendimientos femeninos, impactando positivamente en la disminución de las brechas de género.

Entre las políticas a desarrollar, pueden estar las relacionadas con disminución de la vulnerabilidad de tipo financiero de las mujeres y su grupo familiar, por medio de organismos de apoyo para estimular el mercadeo de bienes y servicios para llegar a otros mercados, acceso a fuentes de crédito, acciones de facilitación para la creación de redes de mujeres emprendedoras, entre otras. De igual forma, brindar asesoría o capacitaciones sobre temas administrativos y financieros, para mejorar la efectividad organizacional. De igual forma, debido a los diferentes roles de las mujeres, organismos de apoyo pueden generar la promoción de esfuerzos emprendedores, dados los beneficios de flexibilizaciones de horarios, superación y desarrollo personal o profesional, entre otras.

\section{Referencias}

Afuah, A. (1999). La dinámica de la innovación organizacional: el nuevo concepto para lograr ventajas competitivas y rentabilidad. México, D.F : Oxford University press México s.a.

Armstrong, P. y Armstrong, H. (1990). Theorizing women's work. Toronto: garamond press.

Barney, J.B. (1991). Firm resources and sustained competitive advantage. Journal of management, 17, 99-120. 
Bates, T. (1985). Entrepreneur human capital endowments and minority business viability. The journal of human resources, 20 (4), 540554 .

Becker, G. (1964). Human capital: a theoretical and empirical analysis, with special reference to education, NY: Columbia university press.

Beltrán, E., Maquieira, V., Álvarez, S. y Sánchez, C. (2001). Feminismos. Debates teóricos contemporáneos. Madrid: Alianza.

Birley, S., Moss, C. y Sanders, P. (1987). Do women entrepreneurs require different training?. American Journal of Small Business, 12 (1), 27-35.

Boden, R.J. y Nucci, A.R. (2000). On the Survival Prospects of Man's and Women's New Business Ventures. Journal of Business Venturing, 15(4), 347-362.

Bosch, E. (2001). Nuevas Estrategias en las Relaciones Hombre-Mujer. Papeles de Cuestiones internacionales, 73, 83-90.

Braidotti, R. (1991). Patterns of Dissonance. London: Polity Press.

Bruderl, J., Preisendörfer, P. y Ziegler, R. (1992). Survival Chances of Newly Founded Business Organizations. American Sociological Review, 57( 2), 227-242.

Buttner, H. y Moore, D. (1997). Women's Organizational Exodus to Entrepreneurship: Self-Reported Motivations and Correlates with Success. Journal of Small Business Management, 25 (2), 34-46.

Cantwell, J. y lammarino, S. (2003). Multinational Corporations and European Regional Systems of Innovation. Londres: Routledge.

Cantwell, J. y Molero, J. (2003). Introduction, en J. Cantwell y J. Molero (comps.). Multinational Enterprises, Innovative Strategies and Systems of Innovation, Londres, Edward Elgar.

Carlsson, B., Jacobsson, S. y otros. (2002). Innovation systems: analytical and methodological issues. Research Policy, 31(2), 34-43.

Casrud, A., Gaglio, C. y Olm, K. (1986). Entrepreneurs, Mentors, Networks, and Successful new venture development. American Journal of Small Business 12 (2), 13-18.

Cliff, J.E. (1998). Does one size fit all? Exploring the relationship between attitudes towards growth, gender, and business size. Journal of Business Venturing, 13 , 523-542.

Conner, K.R. (1991). A historical comparison of resource-based theory and five schools of thought within industrial organization economics: Do we have a new theory of the Firm?. Journal of Management, 17 (1), 121-154.

Cooke, P., Gómez, M. y Etxebarria, G. (1997). Regional innovation systems: institutional and organizational dimension. Research Policy, 26 (4), 57-72.

Corona, L. (2002). Innovación y Competitividad Empresarial. Aportes: revista de la facultad de economía, 20, 24-38.

Craig, S. (1992). Men, Masculinity and the Media. Newbury Park, CA: Sage.

Cuervo, A. (1995). La dirección estratégica de la empresa. En Dirección de Empresas de los Noventa. Homenaje al profesor Marcial-Jesús López Moreno, Madrid: Editorial Civitas.

De Martino, R. y Barbato, R. (2003). Differences between women and men MBA entrepreneurs: exploring family flexibility and wealth creation as career motivators. Journal of Business Venturing, 18 (6), 815 - 832.

Dieguez, M.I., Sinde, A.I. y Gueimonde, A. (2007). Teoría de Recursos y capacidades y teoría del capital Humano: bases teóricas de la formación de recursos humanos como mecanismo de apoyo de las nuevas tecnologías de fabricación. Alta Dirección, Año XLIII, 253, 101-109.

Fasci, M.A. y Valdez, J. (1998). Performance contrast of male- and female-owned small accounting practices. Journal Small Business Management, 36, 1-7.

Fischer, E. M., Reuber, R. A., y Dyke, L. S. (1993). A Theoretical Overview and Extension of Research on Sex, , gender and entrepreneurship. Journal of Business Venturing 8 (2), 151-168. 
Gamero, C. y Lassibille, G. (2008). La Escolarización, Trabajo Infantil Y Satisfacción En El Empleo: Evidencia Para Etiopía. Investigaciones de Economía de la Educación, 3, 1-12.

Gatewood, E.J, Gartner, W.B., y Shaver , K.G. (1995). A Longitudinal Study of Cognitive Factors Influencing Start-Up Behaviours and Success at Venture Creation. Journal of Business Venturing, 10 (5), 11-31.

Gill, I. (1989). Technological change, education and obsolescence of human capital: Some evidence for the U.S. Mimeo, State University of New York at Buffalo.

Grant, J. y Tancred, P. (1992). Feminist perspective on State Bureaucracy en: Gendering Organizational Analysis. Ed Mills . Newbury Park.

Grant, R. M. (1991). The Resource-based Theory of Competitive Advantage: Implications for Strategy Formulation. California Management Review, 33 (3), 114-135.

Guzmán, J y Rodríguez; M. (2008). Comportamiento de las Mujeres Empresarias: Una visión Global. Revista de Economia Mundial 18, 381- 392.

Hall, R. (1992). The strategic analysis of intangible resources. Strategic Management Journal, $13,135-144$.

Howells, J. (1999). Regional systems of innovation?, en d. Archibugi, j. Howells y J. Michie, Innovation Policy in a Global Economy, Cambridge, Cambridge University Press.

Hunt, P (1980). Gender and Class Consciousness. London: Macmillan.

Iammarino, S. (2005). An evolutionary integrated view of regional systems of innovation. Concepts, measures and historical perspectives. European Planning Studies, 13 (4),52-73.

Jöreskog, K.G. (1969). A general approach to confirmatory maximum likelihood factor analysis. Psychometrika, 34, 183-202.

Ljunggren, E. y Kolvereid, L. (1996). New Business Formation: Does Gender Make a Difference?. Women in Management Review, 11(4), 3-12.
Malmberg, A., Sölvell, O. y Zander, I. (1996). Spatial clistering, local accumulation of knowledge and firm competitiveness. Geografiska Annaler, 78 (2), 33-50

Marlow, S. (1997): "Self-Employed Women. New Opportunities, Old Challenges?”, Entrepreneurship \& Regional Development, 9, 199210.

Mertens, I. (2002). Formación, productividad y competencia laboral en las organizaciones: conceptos, metodologías y experiencias. Trazos de la Formación, 15. Montevideo: Cinterfor.

Moore, D.P. y Buttner, E.H. (1997). Women's Organizational Female Business Owners: An Exploratory Study. Journal Small Business Management, July, 18-34.

Murillo, S. (2000). Las relaciones de poder entre hombres y mujeres. Los efectos de aprendizaje de rol en los conflictos y en la violencia de género. Madrid. Federación de Mujeres.

Nelson, R., y Phelps, E., (1996). Investments in Humans, Technological Diffusion and Economic Growth. American Economic Review, 61, 139-162.

Peteraf, M. A. (1993). The Cornerstones of Competitive Advantage: A Resource-based View. Strategic Management Journal, 14, 179-191.

Renzulli, L.A., Aldrich, H. y Moody, J. (2000). Family matters: Gender, networks and entrepreneurial outcomes. Social Forces, 79 (2), 523-546.

Rodeiro, D. y López, M.C., (2007). La innovación como factor clave en la competitividad empresarial: un estudio empírico en pymes. Revista Gallega de Economía, 16 (2), 1-18.

Rumelt, R. P. (1984). Toward a Strategic Theory of the Firm. Resources Firms and Strategic. Editado por Nicolai J. Foss, Oxford University Press, 131-135.

Rumelt, R. P. (1987). Theory, Strategy and Entrepreneurship. Handbook of Entrepreneurship Research, US, 2, 11-32.

Saco, D. (1992). Masculinity as signs: poststructualist feminist approaches to the Study of 
Gender. Men, Masculinity and the Media, ed. Steve Craig, 23-39.

Saurina, C. y Coenders, G. (2002). Predicting overall service quality. A structural equation modelling approach. Developments in Social Science Methodology, Metodološki Zvezki, 18, 217-238.

Scott, J. (1990). Historia y Género: las mujeres en Europa moderna y contemporánea. Valencia: Ediciones Alfons.

Shaktini, N. (1985). Le déplacement du sujet phallique: l'écriture lesbienne de Monique Wittig. Vlasta, 4, 65-77.

Sisson, K. y Storey, J. (2000). Realities of Human Resource Management: Managing the Employment Relationship. Buckingham: Open University Press.

Snell, S. y Dean, J. (1992). Integrated Manufacturing and Human Resource Management: A Human Capital Perspective. Academy of Management Journal, 35 (3), 467-504.

Srinivasan, R., Woo, C. y Cooper, A. (1994). Performance determinants for male and female entrepreneurs. Frontiers of Entrepreneurship Research. Ed. W.D.

Valenzuela, A. (2000). Modelo de capacidades para la internacionalización de la empresa exportadora española. Cuadernos de economía y dirección de la empresa, 5, 91-109.

Ventura, J. (1996). Análisis dinámico de la estrategia empresarial: un ensayo interdisciplinar. España: Servicio de Publicaciones, Universidad de Oviedo.

Wernerfelt, B. (1984). A Resource-Based View Of The Firm. Strategic Management Journal, 5 (2), 171-180. 